\title{
Asymptotics and Hamiltonians in a first-order formalism
}

\author{
Abhay Ashtekar ${ }^{1}$, Jonathan Engle ${ }^{1,2}$ and David Sloan ${ }^{1}$ \\ ${ }^{1}$ Institute for Gravitation and the Cosmos, Penn State, University Park, PA 16802, USA \\ 2 Albert Einstein Institute, Am Mühlenberg 1, 14776 Golm, Germany \\ E-mail: ashtekar@gravity.psu.edu, engle@cpt.univ-mrs.fr and sloan@gravity.psu.edu
}

Received 19 February 2008, in final form 18 March 2008

Published 22 April 2008

Online at stacks.iop.org/CQG/25/095020

\begin{abstract}
We consider four-dimensional spacetimes which are asymptotically flat at spatial infinity and show that, in the first-order framework, the action principle for general relativity is well defined without the need of infinite counter terms. It naturally leads to a covariant phase space in which the Hamiltonians generating asymptotic symmetries provide the total energy-momentum and angular momentum of the spacetime. We address the subtle but important problems that arise because of logarithmic translations and super translations both in the Lagrangian and Hamiltonian frameworks. As a forthcoming paper will show, the treatment of higher dimensions is considerably simpler. Our first-order framework also suggests a new direction for generalizing the spectral action of non-commutative geometry.
\end{abstract}

PACS numbers: $04.20 . \mathrm{Cv}, 04.20 . \mathrm{Ha}, 04.20 . \mathrm{Fy}$

\section{Introduction}

In most field theories the action depends only on fundamental fields and their first derivatives. In contrast, the Einstein-Hilbert action of general relativity depends also on the second derivative of the fundamental field, the spacetime metric $g$. As a consequence, stationary points of this action do not yield Einstein's equations unless both the metric and its first derivatives are kept fixed at the boundary; strictly we do not have a well-defined variational principle. To remedy this situation, Gibbons and Hawking $[1,2]$ proposed that we add a surface term to the Einstein-Hilbert action. We are then led to

$$
S_{\mathrm{EH}+\mathrm{GH}}(g)=\frac{1}{2 \kappa}\left(\int_{\mathcal{M}} R \mathrm{~d}^{4} V+2 \int_{\partial \mathcal{M}} K \mathrm{~d}^{3} V+C\right) .
$$

Here, $\kappa=8 \pi G, \mathcal{M}$ is a 4-manifold representing an appropriate portion of spacetime, $\partial \mathcal{M}$ is its boundary, $R$ is the Ricci scalar of the metric $g, K$ is the trace of the extrinsic curvature of $\partial \mathcal{M}$ and $C$ is an arbitrary function of the metric $h$ induced on $\partial \mathcal{M}$ by $g$. 
Let us restrict ourselves to cases where $g$ has signature,,,-+++ and is smooth and globally hyperbolic. We will let $\mathcal{M}$ be the spacetime region bounded between two Cauchy surfaces. If $\mathcal{M}$ is spatially compact, by setting $C=0$, we obtain a well-defined variational principle and a finite on-shell action. However, in the asymptotically flat case, it is well known that this strategy has some important limitations (see, e.g., [3]). In particular, the action is typically infinite even 'on-shell', and indeed even when $g$ is the Minkowski metric. To remedy this problem, Gibbons and Hawking [1,2] proposed an infinite subtraction: carry out an isometric embedding of $(\partial \mathcal{M}, h)$ in Minkowski space, calculate the trace $K_{o}$ of the extrinsic curvature of $\partial \mathcal{M}$ defined by the Minkowski metric and set $C=-K_{o}$. Can this procedure be carried out for generic asymptotically flat Lorentzian metrics $g$ ? Now, there is a theorem due to Weyl which guarantees that any 2-manifold with a metric of positive scalar curvature can be isometrically embedded in the Euclidean 3-space. One may imagine that this result could be extended to higher dimensional Minkowski spaces. However, this expectation is not borne out. For, in a $d$-dimensional spacetime, the metric $h$ on the boundary $\partial \mathcal{M}$ has $d(d-1) / 2-(d-1)$ degrees of freedom (after removing the diffeomorphism gauge), while the choice of embedding provides a freedom worth only one function on $\partial \mathcal{M}$. Thus, even at this heuristic level, if $d \geqslant 4$ the freedom is not sufficient whence this infinite subtraction procedure will not work for generic metrics $g$.

Over the last few years, a new set of proposals for infinite counter terms $C$ have appeared in the literature, motivated in part by the earlier work of Brown and York [4]. In particular, Kraus, Larsen, Siebelink [5] have constructed a counter term using a (non-polynomial) function of the Ricci curvature of the boundary. Mann and Marolf [3] have introduced a counter term which is closer to the spirit of the Gibbons-Hawking proposal. They replace $K_{o}$ with the trace of a tensor field $\hat{K}_{a b}$ which generalizes the extrinsic curvature $K_{a b}^{o}$ of $\partial \mathcal{M}$ with respect to the Minkowski metric, used by Gibbons and Hawking, to situations in which the boundary may not be isometrically embedable in Minkowski space. The Mann-Marolf procedure is motivated by the form of the Gauss-Codazzi equations and is carried out entirely in the physical spacetime, without recourse to any embedding. Not only do these improved actions $S_{\text {imp }}$ lead to welldefined action principles, but they also overcome another limitation of the original proposal: now $\delta S_{\text {imp }}=0$ at asymptotically flat solutions for all permissible variations $\delta$.

Since we are dealing just with classical field theories where all fields are smooth, one might wonder if there is a way to avoid infinite subtractions altogether and construct an action principle which is manifestly finite from the beginning. The first goal of this paper is to show in some detail that this is indeed possible if one uses a first-order framework based on orthonormal tetrads and Lorentz connections ${ }^{3}$. It is worth noting that this framework constitutes the starting point of Hamiltonian loop quantum gravity as well as spin foams. The second goal of the paper is to use this action to construct a covariant Hamiltonian framework by keeping careful track of boundary conditions ${ }^{4}$. It is well known that the asymptotic structure is quite subtle in four dimensions because of the presence of logarithmic translations and super translations $[10,11]$. These subtleties lead to considerable complications in the definition of asymptotic symmetries and conserved quantities. The ensuing difficulties were overcome in discussions of conserved quantities based on field equations quite some time ago [10-14]. However, in much of the older literature on the derivation of Hamiltonians from action, these subtleties were generally overlooked (see, e.g., [15-17]). We will fill this gap starting with the first-order action principle, thus providing a self-contained Lagrangian and Hamiltonian

3 This fact was used in $[6,7]$ where, however, attention was focused on the inner, isolated horizon boundary rather than at infinity.

4 For a complementary treatment in the canonical framework, see [8, 9]. However, that analysis is based on self-dual, rather than real Lorentz connections and did not address issues involving the action principle discussed above. 
descriptions without the need of any infinite counter terms. The first-order framework also has the technical advantage that calculations can be carried out using exterior calculus and tend to be significantly simpler.

The paper is organized as follows. In section 2 we recall the asymptotic structure at spatial infinity in four dimensions, and in section 3 we introduce the Lagrangian and Hamiltonian frameworks using the first-order framework. A careful choice of boundary conditions eliminates the logarithmic and super translations and reduces the asymptotic symmetry group to Poincaré. In section 4 we calculate the Hamiltonians generating these Poincaré symmetries, and in section 5 we discuss their properties. In particular, although they are now defined using entirely different techniques, these expressions of Poincaré momentum agree with the earlier ones [10, 12-14] obtained using field equations. In this respect, the results of section 5 are similar to those obtained by Mann, Marolf and Virmani $[3,18]$ using their infinite counter-term subtraction in the second-order framework. Section 6 summarizes the results and discusses their relevance to recent developments concerning the spectral action used in non-commutative geometry $[19,20]$.

\section{Asymptotic structure: subtleties in four dimensions}

In this section, we will introduce some notation, specify our boundary conditions at spatial infinity and discuss some important complications that are peculiar to four dimensions.

Let $\eta$ be a Minkowski metric on $\mathbb{R}^{4}$. Since we are primarily interested in spatial infinity, we will focus on the region $\mathcal{R}$ which lies outside the light cone of some point $p$ in the interior. On $\mathcal{R}$ the four-dimensional radial coordinate $\rho$ is given by $\rho^{2}=\eta_{a b} x^{a} x^{b}$, where $x^{a}$ are the Cartesian coordinates of $\eta$ with $p$ as origin. Functions $f$ of interest will admit a power series expansion of the type

$$
f(\rho, \Phi)=\sum_{n=0}^{m} \frac{{ }^{n} f(\Phi)}{\rho^{n}}+o\left(\rho^{-m}\right)
$$

where $\Phi=(\theta, \phi, \chi)$ are the standard angles on hyperboloids $\mathcal{H}$ defined by $\rho=$ const and where the remainder $o\left(\rho^{-m}\right)$ has the property that $\lim _{\rho \rightarrow \infty} \rho^{m} o\left(\rho^{-m}\right)=0$. Such a function will be said to admit an asymptotic expansion to order $m$. A tensor field $T^{a \ldots b}{ }_{c \ldots d}$ will be said to admit an asymptotic expansion to order $m$ if all its components in the Cartesian chart $x^{a}$ do so. The derivatives $\partial T^{a \ldots b}{ }_{c \ldots d} / \partial x^{e}$ of such a tensor field with respect to $x^{e}$ will be assumed to admit an asymptotic expansion to order $m+1$. Finally, note that the remainder in (2.1) can contain terms of the type $(h(\Phi) \ln \rho) / \rho^{m+1}$, whence $\lim _{\rho \rightarrow \infty} \rho^{m+1} o\left(\rho^{-m}\right)$ need not exist.

A smooth spacetime metric $g$ on $\mathcal{R}$ will be said to be weakly asymptotically flat at spatial infinity if there exists a Minkowski metric $\eta$ such that outside a spatially compact worldtube $g-\eta$ admits an asymptotic expansion to order 1 and $\lim _{\rho \rightarrow \infty}(g-\eta)=0$. This condition implies that in the $(\rho, \Phi)$ chart associated with $\eta$, the line element of $g$ can be expanded as follows:

$g_{a b} \mathrm{~d} x^{a} \mathrm{~d} x^{b}=\left(1+\frac{2 \sigma(\Phi)}{\rho}\right) \mathrm{d} \rho^{2}+2 \rho \frac{A_{i}(\Phi)}{\rho} \mathrm{d} \rho \mathrm{d} \Phi^{i}+\rho^{2}\left(h_{i j}+\frac{{ }^{1} h_{i j}}{\rho}\right) \mathrm{d} \Phi^{i} \mathrm{~d} \Phi^{j}+o\left(\rho^{-1}\right)$,

where $h_{i j}$ is the metric on the unit (timelike) hyperboloid in Minkowski space. Given such an expansion, it is tempting to think of $\eta$ as a reference metric, consider its Poincare group as the asymptotic symmetry group of $g$ and define Poincaré momentum-i.e., energy-momentum and relativistic angular momentum-using Hamiltonians generating these transformations on the appropriate phase space. Indeed, this is often done in the literature. However, the 
problem is that the metric $\eta$ is not uniquely singled out by the physical metric $g$. For, if $g$ admits an asymptotic expansion (2.2) with respect to one Minkowski metric $\eta$, it also admits this expansion with respect to another Minkowski metric $\bar{\eta}$ if $\eta-\bar{\eta}$ admits an asymptotic expansion to order 1 and $\lim _{\rho \rightarrow \infty}(\eta-\bar{\eta})=0$. Such Minkowskian metrics will be said to be compatible. One might first think that the Poincare groups of compatible metric would agree asymptotically. Unfortunately, as we now show, this is not the case in four dimensions.

Set

$$
\bar{x}^{a}=L_{a}{ }^{b} x^{b}+\ln \rho C^{a}+T^{a}+S^{a}(\Phi)+o\left(\rho^{0}\right),
$$

where $L_{a}{ }^{b}$ is a Lorentz transformation (i.e. $L_{a}{ }^{b} L_{c}{ }^{d} \eta_{b d}=\eta_{a c}$ ), and $T^{a}, C^{a}$ are constant vector fields with respect to $\eta$. Let $\bar{\eta}^{a b}$ be the Minkowski metric for which $\bar{x}^{a}$ is the Cartesian chart. Then it is easy to check that $\eta$ and $\bar{\eta}$ are compatible. If $C^{a}$ and $S^{a}(\Phi)$ were set to zero, $\bar{x}^{a}$ would be just another Cartesian chart for $\eta$ to leading order, whence Poincaré groups of $\bar{\eta}$ and $\eta$ would have agreed asymptotically. The term $C^{a} \ln \rho$ defines a logarithmic translation, while the angle-dependent translation $S^{a}(\Phi)$ is called a super translation. If $C^{a}$ and $/$ or $S^{a}(\Phi)$ are nonzero, the Poincaré groups of $\eta$ and $\bar{\eta}$ fail to agree even at infinity. Since one can define Poincaré momentum using any of them, there is a considerable ambiguity in the values of conserved quantities. The ambiguity introduced by logarithmic translations was first pointed out by Bergmann [21], while that associated with super translations was first observed at null infinity by Bondi and Sachs [22] and later at spatial infinity in [10]. These complications arise because, for physical reasons, we can only ask that $g-\eta$ should fall off as $\left(\rho^{-1}\right)$ in four dimensions. Had we demanded that they fall off as $\left(\rho^{-2}\right)$, we would have been forced to set $C^{a}$ and $S^{a}(\Phi)$ to zero and the ambiguities would have disappeared. But of course, because of the positive energy theorem, in this case the only solution satisfying our putative boundary conditions would have been Minkowski space! In $d$ spacetime dimensions, the physically correct condition is that $g-\eta$ should fall off as $\left(\rho^{-(d-3)}\right)$, whence there is no freedom to perform super translations or logarithmic translations if $d>4$.

The challenge in four dimensions, then, is to strengthen the boundary conditions so that this freedom is eliminated without excessively limiting permissible spacetime geometries. Let us begin with the logarithmic translations. It is straightforward to verify that under $x^{a} \rightarrow \bar{x}^{a}=\ln \rho C^{a}$ we are led to a Minkowski metric $\bar{\eta}$ and hence the 'barred'-version of expansion (2.2) in which

$$
\bar{\sigma}(\Phi)=\sigma(\Phi)+C^{a} \hat{x}_{a}, \quad \text { where } \quad \hat{x}_{a}=\frac{\eta_{a b} x^{b}}{\rho} .
$$

Note that for any nonzero $C^{a}, C^{a} \hat{x}_{a}$ is a function on the hyperboloids $\mathcal{H}$ which is odd under the reflection $\hat{x}^{a} \rightarrow-\hat{x}^{a}$. Therefore, one can eliminate the freedom to perform the logarithmic translations by simply demanding that in the asymptotic expansion $(2.2), \sigma(\Phi)$ be even under these reflections [11]. How stringent is this requirement on $\sigma$ ? Analysis of the asymptotic structure at spatial infinity at $i^{o}$ ties it to the asymptotic behavior of the 'electric' part $E_{a b}=C_{a c b d} \hat{x}^{c} \hat{x}^{d}$ of the Weyl tensor. The leading asymptotic part of $E_{a b}$ is a tensor field tangential to $\mathcal{H}$ given by

$$
{ }^{3} E_{a b}(\Phi):=\lim _{\rho \rightarrow \infty} \rho^{3} E_{a b}
$$

It admits a scalar potential which is precisely $\sigma:{ }^{3} E_{a b}=-\left(D_{a} D_{b} \sigma+\sigma h_{a b}\right)$, where $D$ is the derivative operator of $\left(\mathcal{H}, h_{a b}\right)$ [12-14]. Now one can show that if ${ }^{3} E_{a b}$ is reflection symmetric, one can always choose a flat metric $\eta$ so that $\sigma$ is also reflection symmetric. Thus, to eliminate logarithmic translations one only needs to ask that ${ }^{3} E_{a b}$ be reflection symmetric and then restrict oneself to asymptotic expansions (2.2) in which $\sigma$ is also reflection symmetric. 
Finally, the symmetry condition on ${ }^{3} E_{a b}$ is not excessively restrictive. For example, it is satisfied if $g$ is asymptotically a finite superposition of Schwarzschild metrics with (possibly) distinct asymptotic time translations [23].

Next, let us consider super translations. We first note a result due to Beig and Schmidt [13]: given a metric $g$ that admits expansion (2.2), one can always find another Minkowski metric (which, for simplicity, we will denote by $\eta$ again) such that the off-diagonal terms vanish to leading order. Thus, in the $(\rho, \Phi)$ chart of the new Minkowski metric, we have

$$
\mathrm{d} s^{2}=\left(1+\frac{2 \sigma}{\rho}\right) \mathrm{d} \rho^{2}+\rho^{2}\left(h_{i j}+\frac{1}{\rho} h_{i j}\right) \mathrm{d} \Phi^{i} \mathrm{~d} \Phi^{j}+o\left(\rho^{-1}\right),
$$

where, by our assumption above, $\sigma(\Phi)$ is reflection symmetric on $\mathcal{H}$. For this metric $g$, one can calculate the asymptotic Weyl curvature which can be decomposed into an electric part and a magnetic part. The leading order magnetic part is a tensor field tangential to $\mathcal{H}$, given by

$$
{ }^{3} B_{a b}=\lim _{\rho \rightarrow \infty}{ }^{\star} C_{a c b d} \hat{x}^{c} \hat{x}^{d} .
$$

${ }^{3} B_{a b}$ admits a natural tensor potential $S_{a b}:={ }^{1} h_{a b}+2 \sigma h_{a b}$ on $\mathcal{H}: B_{a b}=\epsilon_{a}{ }^{m n} D_{m} S_{n b}$, where $\epsilon$ and $D$ are the alternating tensor and the derivative operator on the hyperboloid $\mathcal{H}$ compatible with the metric $h_{a b}$, and indices are raised and lowered by $h_{a b}$. Under super translations, $x^{a} \rightarrow \bar{x}^{a}=x^{a}+S^{a}(\Phi)$, the field ${ }^{3} B_{a b}$ itself is left invariant while the potential transforms non-trivially:

$$
\begin{aligned}
& \bar{\sigma}=\sigma, \quad{ }^{1} \bar{h}_{a b}={ }^{1} h_{a b}-2 D_{a} D_{b} f-2 f h_{a b}, \\
& \text { so that } \quad \bar{S}_{a b}=S_{a b}-2 D_{a} D_{b} f-2 f h_{a b},
\end{aligned}
$$

where the function $f$ on $\mathcal{H}$ is given by $f=S^{a}(\Phi)$. Finally, one can show that if ${ }^{3} B_{a b}$ vanishes, then this transformation property of $S_{a b}$ is sufficient to ensure that (via a suitable super translation) we can always choose $\eta$ such that $S_{a b}=0$, i.e., ${ }^{1} h_{a b}=-2 \sigma h_{a b}[10,12-14]$. The transformation property (2.8) immediately implies that this exhausts the super-translation freedom. The condition ${ }^{3} B_{a b}=0$ is not excessively stringent. For example, it is automatically satisfied if $g$ is stationary or axi-symmetric [24].

Let us summarize. If the asymptotic Weyl curvature of a weakly asymptotically flat spacetime $\left(\mathcal{M}, g_{a b}\right)$ is such that its leading order term is purely electric (i.e. if ${ }^{3} B_{a b}=0$ ) and reflection symmetric on $\mathcal{H}$, then one can eliminate both super translations and logarithmic translations by strengthening the boundary conditions in a natural fashion by requiring in (2.6) that $\sigma$ be reflection symmetric and ${ }^{1} h_{a b}=-2 \sigma h_{a b}$. Thus, the metrics $g$ satisfying these additional conditions can be expanded as

$$
\mathrm{d} s^{2}=\left(1+\frac{2 \sigma}{\rho}\right) \mathrm{d} \rho^{2}+\left(1-\frac{2 \sigma}{\rho}\right) \rho^{2} h_{i j} \mathrm{~d} \Phi^{i} \mathrm{~d} \Phi^{j}+o\left(\rho^{-1}\right),
$$

where $\sigma$ is reflection symmetric. Such spacetimes will be said to be asymptotically flat at spatial infinity. If a given physical metric $g$ satisfies these conditions with respect to two distinct Minkowski metrics $\eta$ and $\bar{\eta}$, then their isometry groups agree to leading order: if $K^{a}$ is a Killing vector of $\eta$, there exists a Killing vector $\bar{K}^{a}$ of $\bar{\eta}$ such that $\lim _{\rho \rightarrow \infty} K^{a}-\bar{K}^{a}=0$. Hamiltonians generating these asymptotic symmetries provide unambiguous definitions of conserved quantities at spatial infinity. We will see that the additional conditions on $\sigma$ and ${ }^{1} h_{a b}$ are essential for surface integral expressions of the Lorentz angular momentum to be well defined.

Thus, although the asymptotic structure is quite subtle in four dimensions, one can strengthen the 'obvious' boundary conditions to eliminate the logarithmic translations and 
super translations and yet admit a very large class of physically interesting examples. (For a treatment of these issues in a canonical framework, see [25].)

\section{Action and the covariant phase space}

We can now construct the Lagrangian and Hamiltonian descriptions in the first-order framework. Our basic gravitational variables will be co-tetrads $e_{a}^{I}$ and Lorentz connections $A_{a}^{I J}$ on spacetime $\mathcal{M}$. Co-tetrads $e$ are 'square-roots' of metrics and the transition from metrics to tetrads is motivated by the fact that tetrads are essential if one is to introduce spinorial matter. $e_{a}^{I}$ is an isomorphism between the tangent space $T_{p}(\mathcal{M})$ at any point $p$ and a fixed internal vector space $V$ equipped with a metric $\eta_{I J}$ with Lorentzian signature. The internal indices can be freely lowered and raised using this fiducial $\eta_{I J}$ and its inverse $\eta^{I J}$. Each co-tetrad defines a spacetime metric by $g_{a b}:=e_{a}^{I} e_{b}^{J} \eta_{I J}$ which also has signature $(-+++)$. Then the co-tetrad $e$ is automatically orthonormal with respect to $g$. Since the connection 1-forms $A$ take values in the Lorentz Lie algebra, $A_{a}^{I J}=-A_{a}^{J I}$. The connection acts only on internal indices and defines a derivative operator

$$
D_{a} k_{I}:=\partial_{a} k_{I}+A_{a I}^{J} k_{J}
$$

where $\partial$ is a fiducial derivative operator which, as usual, will be chosen to be flat and torsion free. As fundamental fields, $e$ and $A$ are independent. However, the equation of motion of $A$ implies that $A$ is compatible with $e$, i.e., is fully determined by $e$. Therefore, boundary conditions on $A$ are motivated by those on $e$. These in turn are dictated by our discussion of asymptotics in section 2 .

In the Lagrangian and Hamiltonian frameworks, we have to first introduce the precise space of dynamical fields of interest. Let us fix, once and for all, a co-frame ${ }^{o} e_{a}^{I}$ such that $g_{a b}^{o}=\eta_{I J}{ }^{o} e_{a}^{I o} e_{b}^{J}$ is flat. The derivative operator defined by ${ }^{o} e_{a}^{I}$ will be denoted by $\partial_{a} ; \partial_{a}{ }^{o} e_{b}^{I}=0$. The Cartesian coordinates $x^{a}$ of $g_{a b}^{o}$ and the associated radial-hyperboloid coordinates $\left(\rho, \Phi^{i}\right)$ will be used in asymptotic expansions. The discussion of section 2 suggests that the co-tetrads $e_{a}^{I}$ should admit an asymptotic expansion of order 1 . This suffices to obtain well-defined 4-momentum. However, detailed analysis shows that to define the Lorentz angular momentum one needs $e_{a}^{I}$ to admit an expansion to order 2 (see section 4). Therefore, we will assume that $e_{a}^{I}$ can be expanded as

$$
e={ }^{o} e(\Phi)+\frac{{ }^{1} e(\Phi)}{\rho}+\frac{{ }^{2} e(\Phi)}{\rho^{2}}+o\left(\rho^{-2}\right),
$$

where ${ }^{1} e_{a}^{I}$ is given by

$$
{ }^{1} e_{a}^{I}=\sigma(\Phi)\left(2 \rho_{a} \rho^{I}-{ }^{o} e_{a}^{I}\right)
$$

with a reflection symmetric $\sigma(\Phi)$ (see (2.9)). Here and in what follows

$$
\rho_{a}=\partial_{a} \rho \quad \text { and } \quad \rho^{I}=\eta^{I J o} e_{J}^{a} \rho_{a} .
$$

To appropriate leading orders, $A_{a}^{I J}$ can be required to be compatible with $e_{a}^{I}$ on the timelike worldtube $\tau_{\infty}$ at spatial infinity which is part of the boundary $\partial \mathcal{M}$ of $\mathcal{M}$. This leads us to require that $A_{a}^{I J}$ is of asymptotic order 3 ,

$$
A={ }^{o} A(\Phi)+\frac{{ }^{1} A(\Phi)}{\rho}+\frac{{ }^{2} A(\phi)}{\rho^{2}}+\frac{{ }^{3} A(\Phi)}{\rho^{3}}+o\left(\rho^{-3}\right) .
$$

Compatibility of $A$ with $e$ and flatness of ${ }^{o} e$ enables us to set ${ }^{0} A={ }^{1} A=0$ and express ${ }^{2} A$ as

$$
\begin{aligned}
{ }^{2} A_{a}^{I J}(\Phi) & =2 \rho^{2} \partial^{[J}\left(\rho^{-11} e_{a}^{I]}\right) \\
& =2 \rho\left(2 \rho^{[I} \rho_{a} \partial^{J]} \sigma-{ }^{o} e_{a}^{[I} \partial^{J]} \sigma-\rho^{-1 o} e_{a}^{[I} \rho^{J]} \sigma\right) .
\end{aligned}
$$

6 
(In spite of the explicit factors of $\rho$, the right side is in fact independent of $\rho$ because $\partial_{a} \sigma \sim \rho^{-1} \times$ (angular derivatives of $\sigma$ ). ) We will not need the corresponding expression of ${ }^{3} A$ in terms of $e$ and therefore demand compatibility between $A$ and $e$ only via (3.5).

\subsection{Action principle}

Consider as before the 4-manifold $\mathcal{M}$ bounded by spacelike surfaces $M_{1}$ and $M_{2}$. We will consider smooth histories $(e, A)$ on $M$ such that $(e, A)$ are asymptotically flat in the sense specified above, and are such that $M_{1}, M_{2}$ are Cauchy surfaces with respect to the spacetime metrics $g$ defined by $e$, and the pull-back of $A$ to $M_{1}, M_{2}$ is determined by the pull-back of $e$. The last condition is motivated by the fact that, since the compatibility between $e$ and $A$ is an equation of motion, boundary values where this compatibility is violated are not of interest to the variational principle. Finally, it is convenient to partially fix the internal gauge on the boundaries. We will fix a constant, timelike internal vector $n^{I}$ so that $\partial_{a} n^{I}=0$ and require that the histories be such that $n^{a}:=n^{I} e_{I}^{a}$ is the unit normal to $M_{1}$ and $M_{2}$.

The first-order gravitational action on these histories is given by (see, e.g., [26])

$$
S(e, A)=-\frac{1}{2 \kappa} \int_{\mathcal{M}} \Sigma^{I J} \wedge F_{I J}+\frac{1}{2 \kappa} \int_{\partial \mathcal{M}} \Sigma^{I J} \wedge A_{I J},
$$

where the 2-forms $\Sigma^{I J}$ are constructed from the co-tetrads and $F$ is the curvature of $A$ :

$$
\Sigma_{I J}:=\frac{1}{2} \epsilon_{I J K L} e^{K} \wedge e^{L} \quad \text { and } \quad F_{I}{ }^{J}=d A_{I}{ }^{J}+A_{I}{ }^{K} \wedge A_{K}{ }^{J} .
$$

As in more familiar field theories, the action now depends only on the fundamental fields and their first derivatives. Although the connection $A$ itself appears in the surface term at infinity, the action is in fact gauge invariant. Indeed, it is not difficult to show that the compatibility between the pull-backs to $M_{1}$ and $M_{2}$ of $e$ and $A$ and the property $\partial_{a} n^{I}=0$ implies that, on boundaries $M_{1}$ and $M_{2}, \Sigma^{I J} \wedge A_{I J}=2 K \epsilon_{a b c}$, where $K$ is the trace of the extrinsic curvature of $M_{1}$ or $M_{2}$ (see, e.g., section 2.3.1 of [27]). Thus, on $M_{1}$ and $M_{2}$, the surface term in (3.6) is precisely the Gibbons-Hawking surface term with $C=0$ in (1.1). Therefore, these surface contributions are clearly gauge invariant. This leaves us with just the surface term at the timelike cylinder $\tau_{\infty}$ at infinity. However, since $e$ has to tend to the fixed co-tetrad ${ }^{o} e$ at infinity, permissible gauge transformations must tend to identity on $\tau_{\infty}$. Since the surface integral on $\tau_{\infty}$ involves only the pull-back of $A$ to $\tau_{\infty}$, it follows immediately that this surface integral is also gauge invariant. Note incidentally that on $\tau_{\infty}$ this term is not equal to the Gibbons-Hawking surface term (because $\partial_{a} \rho^{I}$ falls off only as $1 / \rho$ ). Therefore, even if we were to assume compatibility between $e$ and $A$ everywhere and pass to a second-order action, (3.6) would not reduce to the Gibbons-Hawking action with $C=0$. It is also inequivalent to the Gibbons-Hawking prescription of setting $C=K_{o}$ because (3.6) is well defined without reference to any embedding in flat space.

Our boundary conditions allow us to rewrite this action as

$$
S(e, A)=\frac{1}{2 \kappa} \int_{M} \mathrm{~d} \Sigma \wedge A-\Sigma \wedge A \wedge A .
$$

Boundary conditions also imply that the integrand falls off as $\rho^{-4}$. Since the volume element on any Cauchy slice goes as $\rho^{2} \sin \theta \mathrm{d} \rho \mathrm{d} \theta \mathrm{d} \phi$, the action is manifestly finite even off shell if the two Cauchy surfaces $M_{1}, M_{2}$ are asymptotically time-translated with respect to each other. Such spacetimes $\mathcal{M}$ are referred to as cylindrical slabs. In this subsection, our discussion will be restricted to such spacetimes ${ }^{5}$.

5 If the two Cauchy surfaces bounding $\mathcal{M}$ are asymptotically boosted with respect to one another, $\mathcal{M}$ is called a boosted slab. For a boosted slab there is no guarantee that the action would be finite off shell. (The situation is the 
On the class of histories considered, it is easy to check that the functional derivatives of the action are well defined with respect to both $e$ and $A$. Variation with respect to the connection yields $D \Sigma=0$. This condition implies that the connection $D$ defined by $A$ acts on internal indices in the same way as the unique torsion-free connection $\nabla$ compatible with the co-tetrad (which satisfies $\nabla_{a} e_{b}^{I}=0$ ). When this equation of motion is satisfied, the curvature $F$ is related to the Riemann curvature $R$ of $\nabla$ by

$$
F_{a b}^{I J}=R_{a b}{ }^{c d} e_{c}^{I} e_{d}^{J} .
$$

Varying the action with respect to $e_{a}^{I}$ and taking into account the above relation between curvatures, one obtains Einstein's equations $G_{a b}=0$. The inclusion of matter is straightforward because the standard matter actions contain only first derivatives of fundamental fields without any surface terms and the standard fall-off conditions on matter fields imply that the matter action is finite on cylindrical slabs also off shell.

\subsection{Covariant phase space}

We will now let $\mathcal{M}$ be $\mathbb{R}^{4}$. The covariant phase space $\Gamma$ will consist of smooth, asymptotically flat solutions $(e, A)$ to field equations on $\mathcal{M}$. Thus, in contrast to section $3.1, \mathcal{M}$ is not restricted to be a cylindrical slab nor are the pull-backs of $(e, A)$ fixed on any Cauchy surfaces. Our task is to use action (3.6) to define the symplectic structure $\Omega$ on this $\Gamma$.

Following the standard procedure (see, e.g., [28]), let us perform second variations of the action to associate with each phase space point $(e, A)$ and tangent vectors $\delta_{1} \equiv\left(\delta_{1} e, \delta_{1} A\right)$ and $\delta_{2} \equiv\left(\delta_{2} e, \delta_{2} A\right)$ at that point, a 3 -form $J$ on $\mathcal{M}$, called the symplectic current:

$$
J\left(\gamma ; \delta_{1}, \delta_{2}\right)=-\frac{1}{2 \kappa}\left[\delta_{1} \Sigma^{I J} \wedge \delta_{2} A_{I J}-\delta_{2} \Sigma^{I J} \wedge \delta_{1} A_{I J}\right] .
$$

Using the fact that the fields $(e, A)$ satisfy the field equations and the tangent vectors $\delta_{1}, \delta_{2}$ satisfy the linearized equations of $(e, A)$, one can directly verify that $J\left(\gamma ; \delta_{1}, \delta_{2}\right)$ is closed as guaranteed by the general procedure involving second variations. Let us now consider a portion $\widetilde{\mathcal{M}}$ of $\mathcal{M}$ bounded by two Cauchy surfaces $M_{1}, M_{2}$. These are allowed to be general Cauchy surfaces so $\widetilde{\mathcal{M}}$ may in particular be a cylindrical or a boosted slab in the sense of section 3.1 . Consider now a region $\tilde{\mathcal{R}}$ within $\widetilde{\mathcal{M}}$, bounded by compact portions $\tilde{M}_{1}, \tilde{M}_{2}$ of $M_{1}$ and $M_{2}$ and a timelike cylinder $\tau$ joining $\partial \tilde{M}_{1}$ and $\partial \tilde{M}_{2}$. Since $d J=0$, integrating it over $\tilde{\mathcal{R}}$ one obtains

$$
\int_{\tilde{M}_{1}} J+\int_{\tilde{M}_{2}} J+\int_{\tau} J=0
$$

The idea is to take the limit as $\tau$ expands to the cylinder $\tau_{\infty}$ at infinity. Suppose the first two integrals continue to exist in this limit and the third integral goes to zero. Then, in the limit the sum of the first two terms would vanish and, taking into account orientation signs, we would conclude that $\int_{M} J$ is a 2-form on $\Gamma$ which is independent of the choice of the Cauchy surface $M$. This would be the desired pre-symplectic structure. However, the issue of whether the boundary conditions ensure that the integrals over Cauchy surfaces converge and the flux across $\tau_{\infty}$ vanishes is somewhat delicate and often overlooked in the literature ${ }^{6}$. If either of these properties failed, we would not obtain a well-defined symplectic structure on $\Gamma$.

same in Yang-Mills theory in Minkowski space.) However, when equations of motion are satisfied the action reduces just to the surface term which, because of our boundary conditions, is proportional to $\int_{\tau_{\infty}} \sigma \mathrm{d}^{3} V$. The asymptotic behavior in time of $\sigma$ [23] implies that this integral is finite. Thus the on shell result of [3,18] is recovered in this first-order framework.

6 Furthermore, even when such issues are discussed, one often considers only the restricted action $\Omega\left(\delta, \delta_{V}\right)$ of the pre-symplectic structure $\Omega$, where one of the tangent vectors, $\delta_{V}$, is associated with an asymptotic symmetry $V^{a}$ on $\mathcal{M}$ in the sense discussed in section 4 because, as we will see, it is this restricted action that directly enters the 
Let us first consider the integral over the timelike boundary $\tau$. As $\tau$ tends to $\tau_{\infty}$, the integrand $J_{a b c} \epsilon^{a b c}$ tends to

$$
\lim _{\tau \rightarrow \tau_{\infty}}\left(\delta_{[1} \frac{{ }^{1} \Sigma_{a b}}{\rho}\right) \wedge\left(\delta_{2]} \frac{{ }^{2} A_{c}}{\rho^{2}}\right) \epsilon^{a b c}=\lim _{\tau \rightarrow \tau_{\infty}} \epsilon_{I J K L}{ }^{o} e_{a}^{K}\left(\delta_{[1}^{1} e_{b}^{L}\right)\left(\delta_{2]}{ }^{2} A_{c}^{I J}\right) \rho^{-3} \epsilon^{a b c},
$$

where $\epsilon^{a b c}$ is the metric-compatible 3-form on $\tau$. Since the volume element on $\tau$ goes as $\rho^{3}$, the integral of the symplectic flux over $\tau$ has a well-defined limit. But the key question is if the limit is zero. If not, there would be a leakage of the current $J$ at spatial infinity and the symplectic structure would not be well defined. Let us evaluate this term using expressions (3.2) and (3.5) of ${ }^{1} e$ and ${ }^{2} A$, respectively. Then the second term in (3.10) reduces to

$\epsilon_{I J K L} e_{a}^{K}\left(2 \rho_{b} \rho^{L}-{ }^{o} e_{b}^{L}\right) \delta_{1} \sigma\left(2 \rho^{I} \rho_{c} \partial^{J} \delta_{2} \sigma-{ }^{o} e_{c}^{I} \partial^{J} \delta_{2} \sigma-{ }^{o} e_{c}^{I} \rho^{J} \delta_{2} \sigma\right) \rho^{-3} \epsilon^{a b c}-1 \leftrightarrow 2$.

The term containing $\delta_{1} \sigma \delta_{2} \sigma$ vanishes because of anti-symmetrization, while the remaining terms containing derivatives of $\delta_{2} \sigma$ vanish because the normal $\rho^{c}$ to $\tau$ is contracted either with $\epsilon^{a b c}$ or the derivative of $\sigma$. Thus, our boundary conditions imply that the symplectic flux across $\tau_{\infty}$ vanishes.

The next question is whether the integral over $\tilde{M}_{1}$ (and $\tilde{M}_{2}$ ) continues to be well defined in the limit as we approach $M_{1}$ (respectively $M_{2}$ ). The leading term is again given by the integral of (3.10) over $M_{1}$, the only difference being that $\epsilon^{a b c}$ is now the metric-compatible 3 -form on $M_{1}$. Since the volume element on $M_{1}$ goes as $\rho^{2} \mathrm{~d} \rho \mathrm{d}^{2} \Phi$, power counting argument says that the integral of this leading term can be logarithmically divergent. However, one can again expand out the leading term as (3.11) and show that it in fact vanishes. Since the remaining integrand falls off at least as fast as $1 / \rho^{4}$, the integral over $M$ converges. Thus, because of our boundary conditions, we are led to a well-defined pre-symplectic structure, i.e., a closed 2-form, on $\Gamma$

$$
\Omega\left(\delta_{1}, \delta_{2}\right)=\frac{1}{2 \kappa} \int_{M} \operatorname{Tr}\left[\delta_{1} \Sigma \wedge \delta_{2} A-\delta_{2} \Sigma \wedge \delta_{1} A\right]
$$

where $M$ is any Cauchy surface in $\mathcal{M}$ and trace is taken over the internal indices. $\Omega$ is not a symplectic structure because it is degenerate. The vectors in its kernel represent infinitesimal 'gauge transformations'. The physical phase space is obtained by quotienting $\Gamma$ by gauge transformations and inherits a true symplectic structure from $\Omega$. We will not carry out the quotient however because the calculation of Hamiltonians can be carried out directly on $(\Gamma, \Omega)$.

\section{Generators of asymptotic Poincaré symmetries}

Let $V^{a}$ be a vector field on $\mathcal{M}$ representing an asymptotic Poincaré symmetry, a Killing vector field of one of the flat metrics $\eta_{a b}$ in $\Gamma$. Then at any point $(e, A)$ of $\Gamma$, the pair $\left(\mathcal{L}_{V} e, \mathcal{L}_{V} A\right)$ of fields satisfies the linearized field equations, whence $\delta_{V}:=\left(\mathcal{L}_{V} e, \mathcal{L}_{V} A\right)$ is a vector field on $\Gamma$. (In the definition of the Lie derivative, internal indices are treated as scalars; thus, $\mathcal{L}_{V} e_{a}^{I}=V^{b} \partial_{b} e_{a}^{I}+e_{b}^{I} \partial_{a} V^{b}$.) The question is whether $\delta_{V}$ is a phase space symmetry, i.e., whether it satisfies $\mathcal{L}_{\delta_{V}} \Omega=0$.

Consider the 1 -form $X_{V}$ on $\Gamma$ defined by

$$
X_{V}(\delta)=\Omega\left(\delta, \delta_{V}\right)
$$

discussion of conserved quantities. Typically the 3-forms $\Omega\left(\delta_{1}, \delta_{V}\right)$ on $\mathcal{M}$ have a better asymptotic behavior that the generic 3-forms $\Omega\left(\delta_{1}, \delta_{2}\right)$. However, unless $\Omega\left(\delta_{1}, \delta_{2}\right)$ is well defined for all $\delta_{1}, \delta_{2}$, one does not have a coherent Hamiltonian framework and cannot start constructing conserved quantities. 
$\mathcal{L}_{\delta_{V}} \Omega=0$ on $\Gamma$ if and only if $X_{V}$ is closed, i.e.,

$$
\mathbb{d} X_{V}=0 \text {, }
$$

where $\mathbb{d}$ denotes the exterior derivative on (the infinite dimensional) phase space $\Gamma$. If this is the case then, up to an additive constant, the Hamiltonian is given by

$$
\mathbb{d} H_{V}=X_{V} \text {. }
$$

The constant is determined by requiring that all Hamiltonians generating asymptotic symmetries at the phase space point $\left({ }^{\circ} e, A=0\right)$ corresponding to Minkowski spacetime must vanish. To calculate the right side of (4.1), it is useful to note the Cartan identities

$\mathcal{L}_{V} A=V \cdot F+D(V \cdot A) \quad$ and $\quad \mathcal{L}_{V} \Sigma=V \cdot D \Sigma+D(V \cdot \Sigma)-[(V \cdot A), \Sigma]$.

Using these, the field equations satisfied by $(e, A)$ and the linearized field equations for $\delta$, one obtains the required expression of $X_{V}$ :

$$
X_{V}(\delta):=\Omega\left(\delta, \delta_{V}\right)=-\frac{1}{2 \kappa} \oint_{S_{\infty}} \operatorname{Tr}[(V \cdot A) \delta \Sigma-(V \cdot \Sigma) \wedge \delta A] .
$$

Note that the expression involves integrals only over the 2-sphere boundary $S_{\infty}$ of the Cauchy surface $M$ (i.e., the intersection of $M$ with the hyperboloid $\mathcal{H}$ at infinity); there is no volume term. This is a reflection of the fact that the theory is diffeomorphism invariant.

\subsection{Energy-momentum}

Let us begin by setting $V^{a}=T^{a}$, an infinitesimal asymptotic translation. Since $\delta \Sigma \sim$ $1 / \rho, A \sim 1 / \rho^{2}$ and since the area element of the 2-sphere $S_{\infty}$ at infinity grows as $\rho^{2}$, the first term on the right side of (4.3) vanishes in the limit and we are left with

$$
X_{T}(\delta):=\Omega\left(\delta, \delta_{T}\right)=\frac{1}{2 \kappa} \oint_{S_{\infty}} \operatorname{Tr}[(T \cdot \Sigma) \wedge \delta A]
$$

which is manifestly well defined. Inserting the asymptotic forms of connection (3.5) and tetrad (3.1), we find

$$
X_{T}(\delta)=\frac{2}{\kappa} \oint_{S_{\infty}}\left[\left(\rho_{a} T^{a}\right) n^{b} \partial_{b} \delta \sigma-\delta \sigma\left(n_{a} T^{a}\right)\right] \mathrm{d}^{2} S_{o},
$$

where $n_{b}$ is the unit normal to the 2-sphere $S_{\infty}$ within the hyperboloid $\mathcal{H}$ and $\mathrm{d}^{2} S_{o}$ is the area element of the unit 2-sphere. Since the only dynamical variable in the integrand is $\sigma$, we can pull the $\delta$ out of the integral and obtain the Hamiltonian $H_{T}$ generating the asymptotic translation $T^{a}$ :

$$
H_{T}=\frac{2}{\kappa} \oint_{S_{\infty}}\left[\left(\rho_{a} T^{a}\right) n^{b} \partial_{b} \sigma-\sigma\left(n_{a} T^{a}\right)\right] \mathrm{d}^{2} S_{o} .
$$

Had we selected a translational Killing field $\bar{T}^{a}$ of another flat metric $\bar{\eta}_{a b}$ in our phase space $\Gamma$, we would have obtained the same answer because $\bar{T}^{a}-T^{a}=o\left(\rho^{-1}\right)$.

Taking our transformation to be a unit time-translation which is asymptotically orthogonal to the Cauchy surface $M$ under consideration (and hence to $S_{\infty}$ ), we find the energy to be

$$
E=\frac{2}{\kappa} \oint_{S_{\infty}} \sigma \mathrm{d}^{2} S_{o}
$$

Similarly, if $T^{a}$ is a space-translation which is asymptotically tangential to $M$, we find

$$
\vec{P} \cdot \vec{T}=\frac{2}{\kappa} \oint_{S_{\infty}}\left(\rho_{a} T^{a}\right)\left(n^{b} D_{b} \sigma\right) \mathrm{d}^{2} S_{o},
$$


where $D$ is the derivative operator on the unit hyperboloid $\left(\mathcal{H}, h_{a b}\right)$. Note that $\rho_{a} T^{a}$ are the ' $\ell=1$ ' spherical harmonics on $S_{\infty}$ determined by translations $T^{a}$.

Thus, the energy-momentum is determined directly by $\sigma$. It follows from [10, 12, 13] that $\sigma$ satisfies the hyperbolic equation $D^{a} D_{a} \sigma+3 \sigma=0$ on $\left(\mathcal{H}, h_{a b}\right)$. Thus, its initial data consists of the pair $\left(\sigma, \dot{\sigma}=n^{a} D_{a} \sigma\right)$ on a 2-sphere cross-section of $\mathcal{H}$. Energy is given by the ' $Y_{00}$ ' component of the first piece of this data, while the momentum by the ' $Y_{1 m}$ ' components of the second piece. Finally, recall that the reflection through the origin of Minkowski space induces an isometry on the unit hyperboloid. Therefore, every solution $\sigma$ to the hyperbolic equation can be decomposed into a part $\sigma_{(E)}$ which is even under this reflection and a part $\sigma_{(O)}$ which is odd. Each satisfies the hyperbolic equation separately. Our boundary conditions require that $\sigma$ be even. However it is easy to verify that, even if this condition had not been imposed, only the even part $\sigma_{(E)}$ contributes non-trivially to expressions (4.7) and (4.8) of energy and momentum, respectively.

\subsection{Relativistic angular momentum}

Let us now set $V^{a}=L^{a}$, an infinitesimal asymptotic Lorentz symmetry. For definiteness, we will assume that it is a Lorentz Killing field of $\eta_{a b}:=\eta_{I J} e_{a}^{I o} e_{b}^{J}$ so that it is tangential to the $\rho=$ const hyperboloids $\mathcal{H}$. The question is whether the vector field $\delta_{L}$ on $\Gamma$ is Hamiltonian. Let us begin by examining the 1 -form $X_{L}$ on $\Gamma$. Using (4.3), we have

$$
X_{L}(\delta):=\Omega\left(\delta, \delta_{L}\right)=\frac{1}{2 \kappa} \lim _{\rho \rightarrow \infty} \oint_{S_{\rho}} \operatorname{Tr}[(L \cdot A) \delta \Sigma+(L \cdot \Sigma) \wedge \delta A],
$$

where $S_{\rho}$ is the 2-sphere intersection of the $\rho=$ constant hyperboloid $\mathcal{H}_{\rho}$ with the Cauchy surface $M$ used to evaluate the symplectic structure. Now, as $\rho$ tends to infinity, $\Sigma$ has a well-defined, nonzero limit, $A \sim \rho^{-2}, \delta A \sim \rho^{-2}, \delta \Sigma \sim \rho^{-1}$ and $L \sim \rho$. Therefore, the second term in (4.9) is potentially divergent. Using the asymptotic form (3.5) of $\delta A$, it follows that the second term is proportional to

$$
\lim _{\rho \rightarrow \infty} \oint_{S_{\rho}}\left(\delta \sigma L^{a} n_{a}\right) \rho^{-2} \mathrm{~d}^{2} S
$$

where $n_{a}$ is the unit normal to $S_{\rho}$ within the hyperboloid $\mathcal{H}_{\rho}$ (or equivalently, to the Cauchy surface $M$ ) and $\mathrm{d}^{2} S$ is the volume element on $S_{\rho}$ (which grows as $\rho^{2}$ ). If we were interested in the rotational sub-group of the Lorentz group adapted to $M$, the vector field $L^{a}$ would be tangential to $M$, whence this term would vanish ${ }^{7}$. But for a boost, $L^{a}$ is proportional to $n^{a}$ whence the potential divergence survives. Recall, however, that our boundary conditions require that $\sigma$ be invariant under reflection symmetry. For a Lorentz boost, on the other hand, $L^{a} n_{a}$ is proportional to $Y_{1 m}$, and therefore odd, whence the integral vanishes. Thus, thanks to the parity condition on $\sigma$, the 1 -form $X_{L}$ is well defined on $\Gamma$.

To extract the Hamiltonian from $X_{L}$, we need to pull $\delta$ out of the integral. This is possible because the reflection symmetry again implies that the potentially divergent contribution from ${ }^{2} A_{a}^{I J}$ vanishes. Furthermore, using the asymptotic forms (3.1) and (3.5), it follows that contributions from $\left(L \cdot{ }^{1} \Sigma\right) \wedge \delta^{2} A$ and $\left(L \cdot{ }^{2} A\right) \wedge \delta^{1} \Sigma$ cancel each other. Consequently, $X_{L}=\mathbb{d} H_{L}$, where the Hamiltonian generating the Lorentz transformation $L^{a}$ is given by

$$
H_{L}=-\frac{1}{2 \kappa} \oint_{S_{\infty}} \operatorname{Tr}\left(\hat{L} \cdot{ }^{o} \Sigma\right) \wedge{ }^{3} A,
$$

7 One might first think that since the integral vanishes for all rotations, by changing the Lorentz frame defining the rotation subgroup one would be able to show that the integral vanishes also for boosts. This turns out not to be correct. To handle Lorentz boosts one needs a genuinely stronger asymptotic condition. As discussed below this is provided by the reflection symmetry of $\sigma$ discussed in section 2 . This point is often not realized because much of the literature focuses only on rotational subgroups. See, e.g., $[3,18]$. 
where $\hat{L}^{a}=L^{a} / \rho$ is the Lorentz Killing field on the unit hyperboloid $\left(\mathcal{H}, h_{a b}\right)$. Note that in contrast to the energy-momentum, the angular momentum is not determined by the leading order deviation of $(e, A)$ from the ground state $\left({ }^{o} e, A=0\right)$ but by sub-leading terms.

\section{Relation to the Spi framework}

The boundary conditions we imposed in section 3 to construct the Lagrangian and Hamiltonian frameworks imply that the spacetime admits a conformal completion with conformal factor $\omega=\rho^{-2}$ in which spatial infinity is represented by a single point $i^{o}$. The conformally rescaled metric $\hat{g}_{a b}=\omega^{2} g_{a b}$ can be shown to have the regularity needed in the so-called Spi framework ${ }^{8}[10,12]$. These conditions in turn imply that various physical fields admit a direction-dependent limit as one approaches $i^{o}$ in spacelike directions and can therefore be regarded as smooth fields on the unit hyperboloid $\mathcal{H}_{o}$ in the tangent space at $i^{o}$. The boundary conditions we imposed have been shown to eliminate the logarithmic translations [11] and super translations $[10,12]$ also in the Spi framework, reducing the asymptotic group to the Poincaré group. Using field equations in the physical spacetime, fields on $\mathcal{H}_{o}$ were shown to satisfy certain equations and these were used to define Poincaré momentum in terms of the asymptotic Weyl curvature. The asymptotic field equations made it evident that these quantities are conserved, i.e., independent of the choice of the 2 -sphere cross-section of $\mathcal{H}_{o}$ used in their evaluation.

In section 4, in contrast, we were led to the expressions of Poincaré momenta using Hamiltonian considerations and our final expressions are surface integrals involving asymptotic forms of triads and connections rather than the Weyl curvature. It is natural to compare the underlying assumptions and ask for the relation between these quantities and those obtained in the Spi framework.

\subsection{Energy-momentum}

In the Spi framework, the total energy-momentum $P_{a}$ is a 4-vector in the tangent space at $i^{o}$. Let $T^{a}$ be an asymptotic translation in the physical spacetime. Then it defines a vector $T_{o}^{a}$ at $i^{o}$ and the corresponding component $T_{o}^{a} P_{a}$ of the 4-momentum is given by $[10,12,14]$

$$
T_{o}^{a} P_{a}=-\frac{1}{\kappa} \oint_{S} \mathbf{E}_{a b} \hat{T}_{o}^{b} n^{a} \mathrm{~d}^{2} S
$$

Here, $\mathbf{E}_{a b}$ is the 'electric part' of the asymptotic Weyl curvature, $\hat{T}_{o}^{a}=h_{b}^{a} T^{b}$ is the conformal Killing field on the unit hyperboloid $\left(\mathcal{H}_{o}, h_{a b}\right)$ in the tangent space $T_{i^{o}}$ and $S$ is any 2-sphere cross section of $\mathcal{H}_{o}$. In terms of the physical spacetime fields used in this paper, $\mathbf{E}_{a b}$ is given by $\mathbf{E}_{a b}={ }^{3} E_{a b} \equiv \lim _{\rho \rightarrow \infty} \rho^{3} C_{a m b n} \rho^{m} \rho^{n}$. Thus, as mentioned in section $2,{ }^{3} E_{a b}$ is the leading order electric part of the asymptotic Weyl tensor, where the electric and magnetic decomposition is carried out using a $\rho=$ const foliation.

To relate this $P_{a}$ to that defined in section 4, let us recall that $\sigma$ serves as a scalar potential of ${ }^{3} E_{a b}$ :

$$
{ }^{3} E_{a b}=-\left(D_{a} D_{b} \sigma+\sigma h_{a b}\right) .
$$

Let us choose $S$ to be the intersection of $\mathcal{H}_{o}$ with a spacelike plane in $T_{i^{o}}$ and let $T^{a}$ be unit and orthogonal to this plane. The question then is whether $T_{o}^{a} P_{a}$ equals energy (4.7) using Hamiltonian considerations.

8 Spi stands for spatial infinity and rhymes with scri that represents null infinity. 
The trace-free property of ${ }^{3} E_{a b}$ implies that $\sigma$ satisfies the hyperbolic equation $D^{a} D_{a} \sigma+3 \sigma=0$ and it is straightforward to verify that, on the specific cross-section $S$ of $\mathcal{H}_{o}$ we chose, $D_{a} \hat{T}_{o}^{b}=0$. Therefore on this $S$, we have $E_{a b} \hat{T}_{o}^{a} n^{b}=E_{a b} \hat{T}_{o}^{a} \hat{T}_{o}^{b}=$ $-\hat{T}_{o}^{a} D_{a} \hat{T}_{o}^{b} D_{b} \sigma+\sigma=-(\Delta+2) \sigma$, where $\Delta$ is the Laplacian on $S$. Hence, we have

$$
T^{a} P_{a}=\frac{2}{\kappa} \oint_{S} \sigma \mathrm{d}^{2} S
$$

which agrees with expression (4.7) of energy obtained from Hamiltonian considerations.

Next, let $T^{a}$ be a space-translation tangential to the 3-plane whose intersection with $\mathcal{H}_{o}$ defined $S$. Then $\hat{T}_{o}^{a} n^{b} h_{a b}=0$ and $D_{a} V^{a}=2 V^{a} \rho_{a}$ on $S$ where $\rho_{a}$ is the unit normal to $\mathcal{H}_{o}$. Using the fact that $D_{a} n^{b}$ vanishes on $S$, we obtain

$$
T^{a} P_{a}=\frac{2}{\kappa} \oint_{S}\left(\hat{T}_{o}^{a} \rho_{a}\right)\left(n^{b} D_{b} \sigma\right) \mathrm{d}^{2} S,
$$

which agrees with expression (4.8) of the 3-momentum obtained from Hamiltonian considerations.

Thus, the energy-momentum obtained using Hamiltonian considerations agrees with that obtained using just the asymptotic field equations in the $i^{o}$ framework. A detailed examination shows that in both frameworks one can impose substantially weaker boundary conditions to arrive at this expression of energy-momentum. In particular, one only needs to require that $e$ be of asymptotic order just 1 (rather than 2) and, furthermore, one can drop the requirement that the $1 / \rho^{3}$ part of the asymptotic Weyl curvature be pure electric and reflection symmetric, thereby allowing both logarithmic translations and super translations.

\subsection{Relativistic angular momentum}

The situation with angular momentum is more subtle. To define Lorentz angular momentum one has to get rid of the super-translation ambiguity also in the Spi framework. Indeed, the procedure we followed in section 2 merely mimicked the Spi strategy of requiring that the $1 / \rho^{3}$ contribution to the magnetic part $B_{a b}$ of the physical Weyl tensor should vanish and then setting its tensor potential to zero. However, in striking contrast to what we found in the discussion of Hamiltonians $H_{L}$ in section 4.2, the Spi angular momentum is well defined without having to require that $\sigma$ be reflection symmetric. Indeed, the Spi expression is constructed directly from ${ }^{4} B_{a b}$ - the $1 / \rho^{4}$ part of $B_{a b}$ - and this field is insensitive to logarithmic translations [11]. Furthermore, as we will now show the final Spi expression coincides with expression (4.11) of $H_{L}$. Why then was the parity condition essential in our derivation of (4.11)? It was necessary because, in the Hamiltonian approach, not only should the expression of angular momentum be well defined, but it should also be the generator of asymptotic Lorentz transformations. More precisely, the parity condition is needed to show that the 1 -form $X_{L}$ on the phase space $\Gamma$ is well defined and exact, i.e., that the right side of (4.11) has the interpretation of Hamiltonians generating Lorentz rotations on the gravitational phase space.

In the Spi framework, angular momentum is constructed from the $1 / \rho^{4}$ part of the magnetic Weyl tensor

$$
{ }^{4} B_{a b}:=\lim _{\rho \rightarrow \infty} \rho^{4 \star} C_{a m b n} \rho^{m} \rho^{n},
$$

where, as before, $\rho^{m}$ is the unit normal to the $\rho=$ const hyperboloids. Every Lorentz Killing field $\hat{L}^{a}$ on the unit hyperboloid is of the form $\hat{L}^{a}=F_{o}^{a b} \rho_{b}$, where $F_{o}^{a b}$ is a constant skew tensor in Minkowski space $(\mathcal{M}, \eta)$ and therefore has a dual Lorentz Killing field defined by 
${ }^{\star} \hat{L}^{a}={ }^{\star} F_{o}^{a b} \rho_{b}$. The angular momentum $J_{\hat{L}}$ associated with the Lorentz Killing field $L^{a}$ is defined as

$$
J_{\hat{L}}=\oint_{S}{ }^{4} B^{a b \star} \hat{L}_{a} \epsilon_{b m n} \mathrm{~d} S^{m n},
$$

where as before $S$ is any 2-sphere cross-section of $\mathcal{H}_{o}$ and $\epsilon$ is the volume 3-form on the unit hyperboloid $\left(\mathcal{H}_{o}, h_{a b}\right)$. To relate $J_{\hat{L}}$ with the Hamiltonian $H_{L}$ of (4.11), we first write the Weyl tensor in (5.5) as $C_{a b c d}=F_{a b}{ }^{I J} e_{c I} e_{d J}$ and use the fact that $F_{a b}{ }^{I J}$ is given by $F_{a b}{ }^{I J}=2 \partial_{[a} A_{b]}+\left[A_{a}, A_{b}\right]^{I J}$. Then, by using the asymptotic expansions (3.1) and (3.5), one obtains $^{9}$

$$
\begin{aligned}
J_{\hat{L}} & =-\frac{1}{2 \kappa} \oint_{S} \operatorname{Tr}\left(\hat{L} \cdot{ }^{o} \Sigma\right) \wedge{ }^{3} A \\
& =H_{L}
\end{aligned}
$$

Thus, the angular momentum constructed from asymptotic field equations in the Spi framework agrees with that obtained in this paper from Hamiltonian considerations. It therefore also follows that in an asymptotically flat axi-symmetric spacetime $H_{L}$ reproduces the Komar integral if $L^{a}$ is chosen to be the rotational Killing field, and in an asymptotically flat stationary spacetime, it yields the same angular momentum dipole moment as that constructed from the stationary Killing field [24].

\section{Discussion}

In this paper, we have shown that in the first-order formalism based on co-tetrads and Lorentz connections, the Lagrangian and Hamiltonian frameworks can be constructed without having to introduce an infinite counter-term subtraction in the action. However, since in four spacetime dimensions physical metrics approach the flat metric only as $1 / \rho$ at spatial infinity, the obvious boundary conditions allow one to make super translations and logarithmic translations. The asymptotic symmetry group is then larger than the Poincare group. If one is interested only in energy-momentum, these ambiguities can be ignored because one can still single out a well-defined four-dimensional group of asymptotic translations. To have a well-defined angular momentum, on the other hand, the obvious boundary conditions used in much of the older literature are too naive; they have to be carefully strengthened to reduce the asymptotic symmetry group to the Poincaré group. When this is done, the Hamiltonians generating asymptotic Poincaré transformations provide us with expressions of energy-momentum and Lorentz angular momentum. These agree with the expressions obtained in the Spi framework based on asymptotic field equations $[10,12]$. Therefore, they also agree with conserved quantities defined by other methods in restricted cases with exact symmetries [24].

For simplicity, in this paper we focused on vacuum Einstein's equations. However, the inclusion of standard matter-in particular, scalar, Maxwell and Yang-Mills fields-with standard boundary conditions used in Minkowski space is straightforward. There are no surface terms in the action associated with matter, and the expressions of the Hamiltonians generating asymptotic Poincare transformations are formally the same as the ones we found. In particular, the Hamiltonians consist entirely of 2-sphere surface integrals at spatial infinity and their integrands do not receive any explicit contributions from matter. Matter makes its presence felt through constraint equations which, in response to matter, modify the asymptotic gravitational fields. Finally, in higher dimensions, the asymptotic structure is considerably

9 The calculation is significantly simplified by noting that (3.1) and (3.4) imply that the $1 / \rho^{3}$ part ${ }^{3} B_{a b}$ of $B_{a b}$ vanishes, whence one can retain just the $1 / \rho^{4}$ terms in the expression of ${ }^{4} B_{a b}$ in terms of $A$. 
simpler because the physical metric approaches the flat metric as $1 / \rho^{2}$ or faster. This issue will be discussed in a future paper [29].

We conclude with a comment. In non-commutative geometry, in place of the Riemannian geometry, one introduces a spectral triplet $(\mathcal{A}, \mathcal{H}, D)$ consisting of a non-commutative $C^{\star}$ algebra $\mathcal{A}$, a representation of it on a Hilbert space $\mathcal{H}$ and a Dirac operator $D$ acting on $\mathcal{H}$. A certain choice of the triplet is made to describe (a generalization of) the standard model of particle physics together with Einstein gravity. Rather general symmetry considerations then lead to a so-called 'spectral action' from which dynamics can be derived [19]. It has been known for some time that an asymptotic expansion of this action can be performed to make contact with the low-energy physics and the first terms reproduce the Einstein-Hilbert action with a cosmological constant. Recently, it was realized [20] that the spectral action can be naturally extended to incorporate the presence of boundaries, and the asymptotic expansion of the new action produces precisely the Einstein-Hilbert action with the Gibbons-Hawking counter term for $C=0$ (see (1.1)). This is an exciting development. However, as the discussion of section 1 shows, in the asymptotically flat context this action has severe limitations and the extension of the spectral action to incorporate the boundary term [20] was motivated using precisely the Hamiltonian formulation in the asymptotically flat context. More generally, the non-commutative framework has been developed primarily for the Riemannian signature and passage to the Lorentz signature is contemplated via a Wick transform in the asymptotically flat context. Therefore, asymptotic considerations of [3] and this paper are directly relevant to the spectral action approach. The natural question then is: can the spectral action framework be further generalized so that the leading terms in the asymptotic expansion yields an action which is free from the drawbacks of (1.1)? The first-order framework discussed in this paper presents a natural avenue for such a generalization. Indeed, the gravitational sector of the noncommutative geometry requires a spin-bundle-and hence a frame field $e$ - as well a Dirac operator-i.e., a spin connection $A$. However, in the non-commutative framework, the two are in essence compatible with one another from the beginning. The question is whether one can extend the framework so that they are independent to begin with and made compatible only by the equations of motion. The spectral action in such a generalization could then descend to (3.6) upon a suitable asymptotic expansion. Quite apart from this specific application, such a 'first-order' framework in non-commutative geometry appears also to lead to mathematical structures which are interesting in their own right, and could provide a technical bridge between non-commutative geometry and loop quantum gravity.

\section{Acknowledgments}

We would like to thank Don Marolf and Nigel Higson for stimulating discussions. This work was supported in part by the NSF grants PHY-0456913 and OISE-0601844, the Alexander von Humboldt Foundation and the Eberly research funds of Penn State.

\section{References}

[1] Gibbons G and Hawking S W 1997 Action integrals and partition function in quantum gravity Phys. Rev. D 152752

[2] Hawking S W 1979 The path integral approach to quantum gravity General Relativity, an Einstein Centenary Survey ed S W Hawking and W Israel (Cambridge: Cambridge University Press)

[3] Mann R B and Marolf D M 2006 Holographic renormalization of asymptotically flat spacetimes Class. Quantum Grav. 2329277 (Preprint hep-th/0511096)

[4] Brown J D and York J W 1993 Quasi-local energy and conserved charges derived from the gravitational action Phys. Rev. D 471407 
[5] Kraus P, Larsen F and Siebelink R 1999 The gravitational action in asymptotically AdS and flat spacetimes Nucl. Phys. B 563259 (Preprint hep-th/9906127)

[6] Ashtekar A, Fairhurst S and Krishnan B 2000 Isolated horizons: Hamiltonian evolution and the first law Phys. Rev. D 62104025 (Preprint gr-qc/0005083)

[7] Ashtekar A, Beetle C and Lewandowski J 2001 Mechanics of rotating isolated horizons Phys. Rev. D 64044016 (Preprint gr-qc/0103026)

[8] Ashtekar A 1987 A new Hamiltonian formulation of general relativity Phys. Rev. D 361587

[9] Thiemann T 1995 Generalized boundary conditions for general relativity for the asymptotically flat case in terms of Ashtekar variables Class. Quantum Grav. 12181 (Preprint gr-qc/9910008)

[10] Ashtekar A and Hansen R O 1978 A unified treatment of spatial and null infinity: I. Universal structure, asymptotic symmetries and conserved quantities at spatial infinity J. Math. Phys. 191542

[11] Ashtekar A 1985 Logarithmic ambiguities in the description of spatial infinity Found. Phys. 15419

[12] Ashtekar A 1980 Asymptotic structure of the gravitational field at spatial infinity General Relativity and Gravitation: One Hundred Years After the Birth of Albert Einstein ed A Held (New York: Plenum)

[13] Beig R and Schmidt B G 1982 Einstein's equations near spatial infinity Commun. Math. Phys. 87 65-80

[14] Ashtekar A and Romano J D 1992 Spatial infinity as a boundary of spacetime Class. Quantum Grav. 9 1069-100

[15] Hawking S W and Horowitz G 1996 The gravitational Hamiltonian, action, entropy and surface terms Class. Quantum Grav. 131487 (Preprint gr-qc/9501014)

[16] Arnowitt R, Deser S and Misner C M 1962 The dynamics of general relativity Gravitation: An Introduction to Current Research ed L Witten (New York: Wiley)

[17] Regge T and Teitelboim C 1974 Role of surface integrals in the Hamiltonian formulation of general relativity Ann. Phys. 88286

[18] Mann R B, Marolf D M and Virmani A 2006 Covariant counterterms and conserved charges in asymptotically flat spacetimes Class. Quantum Grav. 23 6357-78

[19] Chamseddine A H and Connes A 1996 A universal action formula Phys. Rev. Lett. $774868-71$ Chamseddine A H and Connes A 1997 Commun. Math. Phys. 186 731-50

[20] Chamseddine A H and Connes A 2007 Quantum gravity boundary terms from spectral action Phys. Rev. Lett. 99071302

[21] Bergmann P G 1961 Gauge invariant variables in general relativity Phys. Rev. 124274

[22] Bondi H, van der Burg M G J and Metzner A W K 1962 Proc. R. Soc. Lond. A 26921

[23] Ashtekar A and Penrose R 1995 Mass positivity from focusing and structure of space-like infinity, Twistor Newsletter 31 in Further Advances in Twistor Theory vol 2 ed L J Mason, L P Hughston and P Z Kobak (New York: Longman)

[24] Ashtekar A and Magnon A 1979 On conserved quantities in general relativity J. Math. Phys. 20793

[25] Beig B and O'Murchadha N 1987 Poincaré group as the symmetry group of canonical general relativity Ann. Phys., NY 174 463-98

[26] Ashtekar A 1991 Lectures on non-perturbative canonical gravity Notes Prepared in Collaboration with $R$ Tate (Singapore: World Scientific)

[27] Ashtekar A and Lewandowski J 2004 Background independent quantum gravity: a status report Class. Quantum Grav. 21 R53-152

[28] Ashtekar A, Bombelli L and Reula O 1991 The covariant phase space of asymptotically flat gravitational fields Analysis, Geometry and Mechanics: 200 Years After Lagrange ed M Francaviglia and D Holm (Amsterdam: North-Holland)

[29] Ashtekar A and Sloan D 2008 Asymptotics and Hamiltonians in a first order formalism (in preparation) 